\title{
A Lifting Method for Krause's Consensus Model
}

\author{
Ningbo Guo ${ }^{1}$, Yaming Chen ${ }^{2, *}$ and Xiaogang Deng ${ }^{2,3}$ \\ ${ }^{1}$ College of Computer, National University of Defense Technology, Changsha, \\ Hunan 410073, P.R. China. \\ ${ }^{2}$ College of Aerospace Science and Engineering, National University of \\ Defense Technology, Changsha, Hunan 410073, P.R. China. \\ ${ }^{3}$ Chinese Academy of Military Science, Beijing 100071, P.R. China.
}

Received 26 April 2021; Accepted 28 September 2021

\begin{abstract}
In this work, we aim to show how to solve the continuous-time and continuous-space Krause model by using high-order finite difference (FD) schemes. Since the considered model admits solutions with $\delta$-singularities, the FD method cannot be applied directly. To deal with the annoying $\delta$-singularities, we propose to lift the solution space by introducing a splitting method, such that the $\delta$-singularities in one spatial direction become step functions with discontinuities. Thus the traditional shock-capturing FD schemes can be applied directly. In particular, we focus on the two-dimensional case and apply a fifthorder weighted nonlinear compact scheme (WCNS) to illustrate the validity of the proposed method. Some technical details for implementation are also presented. Numerical results show that the proposed method can capture $\delta$ singularities well, and the obtained number of delta peaks agrees with the theoretical prediction in the literature.
\end{abstract}

AMS subject classifications: 65M06, 35L65, 35L81

Key words: Krause's consensus model, lifting method, finite difference method, $\delta$-singularities.

\footnotetext{
*Corresponding author. Email address: chenym-08@163.com (Y. Chen)
} 


\section{Introduction}

It is believed that the finite difference (FD) method cannot be applied directly for solving problems with $\delta$-singularities appearing in the solutions. For problems with $\delta$-singularities entering initial value conditions or source terms, one may regularize the Dirac delta function by using a nonsingular function (see [17] and references therein), and then apply the traditional FD method as usual. However, these regularizations may smear the solutions severely and lead to large errors in the approximation. In addition, this approach would encounter difficulty when $\delta$-singularities arise in the solution as time goes on. Krause's model in continuous form is typically one of the models that $\delta$-singularities may appear in the solution for long time, even for a smooth initial condition. Moreover, the number of delta peaks and the positions vary rapidly for different definitions of the velocity. Thus, it is hard to construct a stable FD scheme for this model.

Krause's model in discrete form has been widely used to analyze the dynamics of multiagent consensus, which has important applications in many disciplines, such as control theory [6] and information engineering [1]. In applications, people are often concerned about the case with a large number of agents in high dimensions. However, it is difficult to analyze the behavior of this case in a discrete framework. As a fundamental step, it is worth studying the dynamical models for continuous distributions of agents; see $[2,5,18]$ for examples.

The so-called continuous-time and continuous-space Krause model presented in [5] reads as

$$
\rho_{t}+\operatorname{div}(V \rho)=0,
$$

where $\rho$ represents the density and $V=(u, v, w, \ldots)$ the velocity that depends on $\rho$ in a nonlinear way

$$
V(\boldsymbol{x}, t)=\int_{B_{R}(x)}(\boldsymbol{y}-\boldsymbol{x}) \rho(\boldsymbol{y}, t) \mathrm{d} \boldsymbol{y} .
$$

Here the integral domain $B_{R}(x)$ is the closed ball

$$
B_{R}(\boldsymbol{x})=\{\boldsymbol{y} \mid\|\boldsymbol{y}-\boldsymbol{x}\| \leq R\},
$$

where $x$ represents a spatial vector, the norm $\|\cdot\|$ can be the Euclidean norm or the maximum norm, and $R$ is the radius of the ball. For simplicity of the computational domain, only the maximum norm is considered in this work.

Since $\delta$-singularities may appear in the solution of Eq. (1.1), it is not surprised that the widely used FD method $[7,8,15,21]$ cannot be applied directly, especially for high-dimensional cases. Thus some authors paid their attention to the finite volume (FV) method [4,5] and discontinuous Galerkin (DG) method [12, 19, 20], 
which are both based on the integral form of the considered equation (1.1). However, the FD method can be implemented in a dimension-by-dimension manner, thus the computational cost for high dimensions can be saved a lot compared to the FV method or the DG method with the same order of accuracy. Therefore, it is worth asking the question that whether there is a way such that one can use the FD method to successfully solve Krause's model with $\delta$-singularity solutions. To answer this, we provide in the present work a novel splitting method to lift the solution space of the model. Thus the annoying $\delta$-singularities in one spatial direction would transform into step functions, which can be successfully simulated by some shock-capturing FD schemes that are widely used for solving hyperbolic conservation laws $[3,10,13]$. It should be mentioned that the validity of the lifting method for the one-dimensional case has been demonstrated in the conference paper [11]. Here for high-dimension cases, we confine the study to the two-dimensional case to illustrate the idea. But we shall note that the extension of the proposed method to higher dimensions is straightforward.

The rest of this work is organized as follows. In Section 2, we present a novel splitting method to lift the solution space of the two-dimensional Krause model and give the details of the numerical method. In Section 3, numerical experiments are performed to demonstrate the validity of the proposed method. Finally, conclusions are drawn in Section 4.

\section{Numerical method}

Motivated by the equivalence between one-dimensional conservation laws and Hamilton-Jacobi equations [14], we may lift the solution space of Eq. (1.1) to avoid dealing with $\delta$-singularities directly. In one dimension, one can introduce $\varphi(x, t)$ such that $\varphi_{x}=\rho$. Then Eq. (1.1) can be obtained by differentiating the following equation:

$$
\varphi_{t}+u \varphi_{x}=0
$$

once with respect to $x$. However, this cannot be extended directly to high dimensions. Instead, to lift the solution space, we need to introduce a vector $\Phi$ such that $\operatorname{div} \Phi=\rho$. For the two-dimensional case, we denote $\Phi=[\varphi, \psi]^{\mathrm{T}}$. Then Eq. (1.1) can be lifted to be

$$
\begin{aligned}
& \varphi_{t}+u\left(\varphi_{x}+\psi_{y}\right)=0, \\
& \psi_{t}+v\left(\varphi_{x}+\psi_{y}\right)=0 .
\end{aligned}
$$

It is noted that Eq. (1.1) can be recovered by adding up the equations obtained by differentiating Eqs. (2.2) and (2.3) with regards to $x$ and $y$, respectively. To see the 
properties of the lifted equations clearly, it is better to write them into a vector form

$$
\Phi_{t}+A \Phi_{x}+B \Phi_{y}=0
$$

where the coefficient matrices

$$
A=\left[\begin{array}{ll}
u & 0 \\
v & 0
\end{array}\right], \quad B=\left[\begin{array}{ll}
0 & u \\
0 & v
\end{array}\right] .
$$

Obviously, the system (2.4) is still hyperbolic, as Krause's model (1.1). Like the one-dimensional case, we expect that $\delta$-singularities in $\rho$ would be regularized in the lifting space. Therefore, the traditional shock-capturing schemes can be applied directly without difficulty. As mentioned, we only consider FD schemes in this current work. Particularly, we confine the study to an explicit fifth-order weighted compact nonlinear scheme (WCNS) [10], which has been applied successfully in the field of computational fluid dynamics [9].

For the considered FD method, it is sufficient to introduce the algorithm for the one-dimensional case. The method is based on a mesh that consists of solution points $x_{j}$ and flux points $x_{j+1 / 2}$. If we divide the considered computational domain $\left[x_{l}, x_{r}\right]$ equally into $N$ cells with length $\Delta x$, then

$$
\begin{cases}x_{j+\frac{1}{2}}=x_{l}+(j-1) \Delta x, & 0 \leq j \leq N \\ x_{j}=x_{l}+\left(j-\frac{1}{2}\right) \Delta x, & 1 \leq j \leq N .\end{cases}
$$

The procedure of the scheme can be summarized as the following three steps:

(i) Interpolate the solution values $\varphi_{j}$ to get left and right values at flux points $x_{j+1 / 2}$, denoted by $\varphi_{j+1 / 2}^{-}$and $\varphi_{j+1 / 2}^{+}$, respectively;

(ii) Compute the left- and right-biased derivatives (denoted by $\left(\varphi_{x}\right)_{j}^{ \pm}$) by using a central difference scheme;

(iii) Solve the obtained semi-discretized system

$$
\frac{\mathrm{d} \varphi_{j}}{\mathrm{~d} t}+\frac{1}{2}\left(u_{j}+\left|u_{j}\right|\right)\left(\varphi_{x}\right)_{j}^{-}+\frac{1}{2}\left(u_{j}-\left|u_{j}\right|\right)\left(\varphi_{x}\right)_{j}^{+}=0
$$

by employing some time-marching schemes, where we have split the flux of Eq. (1.1) into two terms according to the upwind properties. 
For step (i), a fifth-order nonlinear interpolation scheme presented in [10] is applied directly. For instance, the value of $\varphi_{j+1 / 2}^{-}$is obtained by using the left-biased stencil $\left\{\varphi_{j-2}, \varphi_{j-1}, \ldots, \varphi_{j+2}\right\}$. To avoid interpolation acrossing discontinuities, the idea is to first interpolate the values $\varphi_{j+1 / 2}^{(k)}$ linearly by using the three-point stencils $\left\{\varphi_{j+k-2}, \varphi_{j+k-1}, \varphi_{j+k}\right\}, k=0,1,2$. Then the recipe of the WENO scheme [13] is adopted to obtain

$$
\varphi_{j+\frac{1}{2}}^{-}=\sum_{k=0}^{2} \omega_{k} \varphi_{j+\frac{1}{2}}^{(k)}
$$

where the details about constructing the nonlinear weights $\omega_{k}$ can be found in [10]. Similarly, based on the right-biased stencil $\left\{\varphi_{j-1}, \varphi_{j}, \ldots, \varphi_{j+3}\right\}$, we can obtain the value of $\varphi_{j+1 / 2}^{+}$straightforwardly. For step (ii), a sixth-order central difference scheme is adopted, i.e.,

$$
\left(\varphi_{x}\right)_{j}^{ \pm}=\frac{1}{\Delta x} \sum_{k=1}^{3} a_{k}\left(\varphi_{j+k-\frac{1}{2}}^{ \pm}-\varphi_{j-k+\frac{1}{2}}^{ \pm}\right)
$$

with the coefficients $a_{1}=75 / 64, a_{2}=-25 / 384$ and $a_{3}=3 / 640$. For step (iii), the third-order strong stability preserving (SSP) Runge-Kutta scheme [16] is employed. For an ordinary differential system $\frac{\mathrm{d}}{\mathrm{d} t} \boldsymbol{\Phi}=R(\boldsymbol{\Phi})$ with $\boldsymbol{\Phi}=\left(\varphi_{1}, \varphi_{2}, \ldots, \varphi_{N}\right)^{\mathrm{T}}$, this time-marching scheme can be expressed as

$$
\left\{\begin{array}{l}
\boldsymbol{\Phi}^{(1)}=\boldsymbol{\Phi}^{n}+\Delta t R\left(\boldsymbol{\Phi}^{n}\right), \\
\boldsymbol{\Phi}^{(2)}=\frac{3}{4} \boldsymbol{\Phi}^{n}+\frac{1}{4}\left[\boldsymbol{\Phi}^{(1)}+\Delta t R\left(\boldsymbol{\Phi}^{(1)}\right)\right], \\
\boldsymbol{\Phi}^{n+1}=\frac{1}{3} \boldsymbol{\Phi}^{n}+\frac{2}{3}\left[\boldsymbol{\Phi}^{(2)}+\Delta t R\left(\boldsymbol{\Phi}^{(2)}\right)\right],
\end{array}\right.
$$

where $\Delta t$ represents the time step.

It should be noted that in our case the velocity $u$ depends on $\rho$ (see Eq. (1.2)). Thus we still need to determine the values of $u_{j}$ before we can solve Eq. (2.7). For the reason of symmetry, we choose here a sixth-order central nonlinear interpolation scheme and compute the term $\rho_{j}=\left(\varphi_{x}\right)_{j}$ by employing the difference scheme (2.9). Then we evaluate the value of $u_{j}$ by using a mid-point rule for the integral (1.2), where we sum over the products of the value of the integrand at cell center and its corresponding cell length. The used nonlinear interpolation scheme is just a straightforward extension of the aforementioned fifth-order interpolation. The only difference is that the central stencil $\left\{\varphi_{j-2}, \varphi_{j-1}, \ldots, \varphi_{j+3}\right\}$ is adopted to compute $\varphi_{j+1 / 2}$, rather than using a biased one. Similarly, based on 
the four three-point stencils $\left\{\varphi_{j+k-2}, \varphi_{j+k-1}, \varphi_{j+k}\right\}, k=0,1,2,3$, linear interpolation schemes are used to get the values $\varphi_{j+1 / 2}^{(k)}$. Then the required value is obtained by

$$
\varphi_{j+\frac{1}{2}}=\sum_{k=0}^{3} \omega_{k} \varphi_{j+\frac{1}{2}}^{(k)}
$$

where the nonlinear weights $\omega_{k}$ are defined exactly in the same way as WCNS, thus the details are also omitted here. Additionally, it should also be mentioned that our final target is to obtain the value of the density $\rho$ rather than $\varphi$, thus the values $\rho_{j}$ obtained by the introduced central scheme is also used to produce the numerical solution of Eq. (1.1).

To implement the introduced scheme for the two-dimensional case (2.4), a similar splitting like Eq. (2.7) is also needed. For the coefficient matrix $A$ defined in Eq. (2.5), it can be diagonalized in the form of $A=P \Lambda P^{-1}$, where

$$
P=\left[\begin{array}{cc}
0 & u / v \\
1 & 1
\end{array}\right], \quad \Lambda=\left[\begin{array}{ll}
0 & 0 \\
0 & u
\end{array}\right] .
$$

Then by decomposing the diagonal matrix as

$$
\Lambda=\left[\begin{array}{cc}
0 & 0 \\
0 & (u+|u|) / 2
\end{array}\right]+\left[\begin{array}{cc}
0 & 0 \\
0 & (u-|u|) / 2
\end{array}\right],
$$

we split the coefficient matrix to be $A=A^{+}+A^{-}$with

$$
A^{ \pm}=\left[\begin{array}{cc}
u \pm|u| & 0 \\
v(1 \pm \operatorname{sign}(u)) & 0
\end{array}\right]
$$

such that $A^{+}$has only nonnegative eigenvalues and $A^{-}$has only nonpositive eigenvalues. By using the same procedure, we can split the coefficient matrix $B$ into $B=B^{+}+B^{-}$with

$$
B^{ \pm}=\left[\begin{array}{cc}
0 & u(1 \pm \operatorname{sign}(v)) \\
0 & v \pm|v|
\end{array}\right]
$$

where the properties of the eigenvalues of $B^{ \pm}$have the same meanings of $A^{ \pm}$. As in the one-dimensional case for computing $\rho_{j}=\left(\varphi_{x}\right)_{j}$, at each solution point $\left(x_{j}, y_{k}\right)$ we first have to use the stated central scheme to evaluate $\rho_{j, k}=\left(\varphi_{x}\right)_{j, k}+$ $\left(\psi_{y}\right)_{j, k}$, and then obtain the values of $u_{i, j}$ and $v_{i, j}$ by using the mid-point rule to evaluate the integral (1.2). Therefore, the splitting matrices at each solution point are determined, denoted by $A_{j, k}^{ \pm}$and $B_{j, k}^{ \pm}$, respectively. Now, according to the upwind-biased property, we obtain a semi-discretized system for Eq. (2.4)

$$
\frac{\mathrm{d} \Phi_{j, k}}{\mathrm{~d} t}+A^{+}\left(\Phi_{x}\right)_{j, k}^{-}+A^{-}\left(\Phi_{x}\right)_{j, k}^{+}+B^{+}\left(\Phi_{y}\right)_{j, k}^{-}+B^{-}\left(\Phi_{y}\right)_{j, k}^{+}=0,
$$


which can be handled by the third-order SSP Runge-Kutta scheme (2.10).

\section{Numerical experiments}

To validate the introduced method, we follow [5] to set the initial condition of Eq. (1.1) to be

$$
\rho(x, y, 0)= \begin{cases}1, & \|x\|<0.5 \\ 0, & \text { otherwise }\end{cases}
$$

where $\|x\|=\max (|x|,|y|)$. However, we need to get the initial conditions of $\varphi$ and $\psi$ rather than $\rho$ to start the computation of the semi-discretized system (2.16). Since we only need to ensure that $\varphi_{x}+\psi_{y}=\rho$, there are many ways to define the initial conditions of $\varphi$ and $\psi$. For the purpose of simplicity and symmetry, we just set

$$
\begin{aligned}
& \varphi(x, y, 0)=\left\{\begin{array}{lll}
0.5 x+0.25, & |x| \leq 0.5, & |y| \leq 0.5 \\
0.5, & x>0.5, & |y| \leq 0.5 \\
0, & \text { otherwise }
\end{array}\right. \\
& \psi(x, y, 0)= \begin{cases}0.5 y+0.25, & |x| \leq 0.5, \quad|y| \leq 0.5 \\
0.5, & |x| \leq 0.5, \quad y>0.5 \\
0, & \text { otherwise }\end{cases}
\end{aligned}
$$

such that

$$
\varphi_{x}(x, y, 0)=\psi_{y}(x, y, 0)=\frac{\rho(x, y, 0)}{2} .
$$

Here, the computational domain is set to be $[-1,1] \times[-1,1]$, i.e., $\|x\| \leq 1$, where the method of extrapolation is used to deal with the boundaries. All results are obtained by using $200 \times 200$ grid points. The time step is set to be $\Delta t=$ $0.1 \min (\Delta x, \Delta y)$ to implement the SSP Runge-Kutta scheme (2.10), where $\Delta x$ and $\Delta y$ denote the spatial steps in the $x$ and $y$ directions, respectively. As shown in Fig. $1, \delta$-singularities are observed clearly in the solutions at time $t=1000$. For different values of the radius $R$, the number of delta peaks varies. The complexity of the solutions increases with the decrease of the values of $R$. Specially, for the cases with $R=0.15$ and $R=0.1$, we see that the height of delta peaks may be different at different positions, but it keeps in a symmetric form with respect to the center of the computational domain. To see more clearly the number and positions of delta peaks, it is better to have a look at the contour plots as depicted in Fig. 2. For the consider four cases with different values $R=0.3,0.2,0.15$ and 0.1 , we see that there are one-delta peak, four-delta peaks, nine-delta peaks and sixteen-delta 

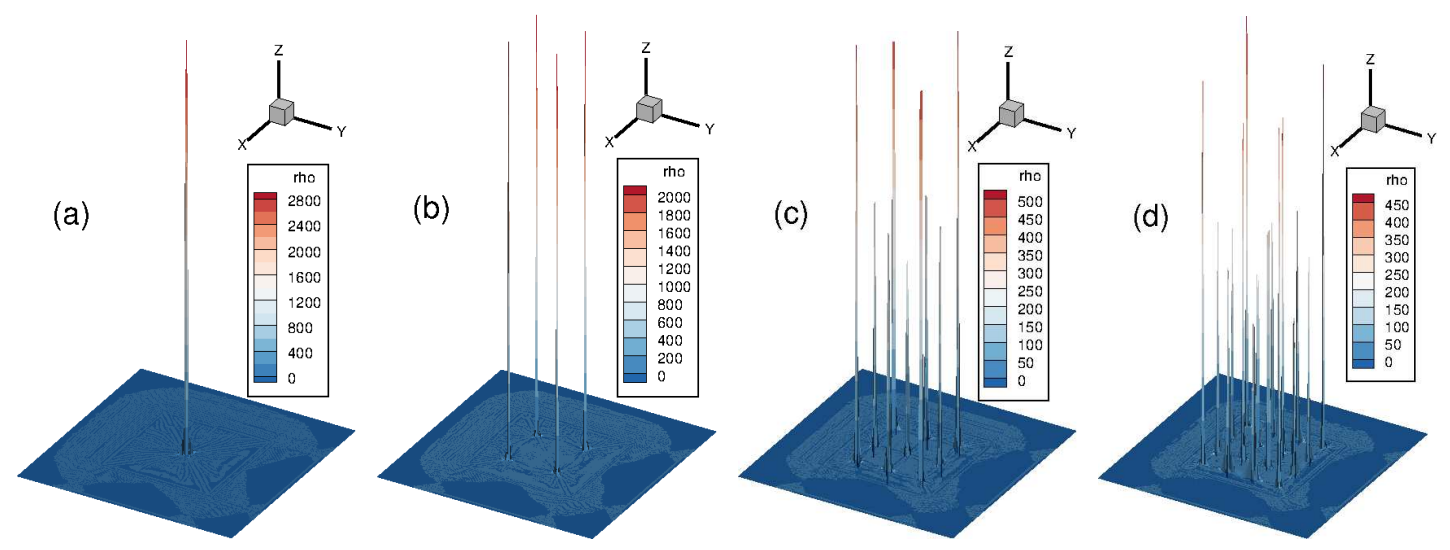

Figure 1: Three-dimensional plots of the numerical results at time $t=1000$ with different values of $R$. (a) $R=0.3$; (b) $R=0.2$; (c) $R=0.15$; (d) $R=0.1$. Here $200 \times 200$ grid points are used to produce the results.
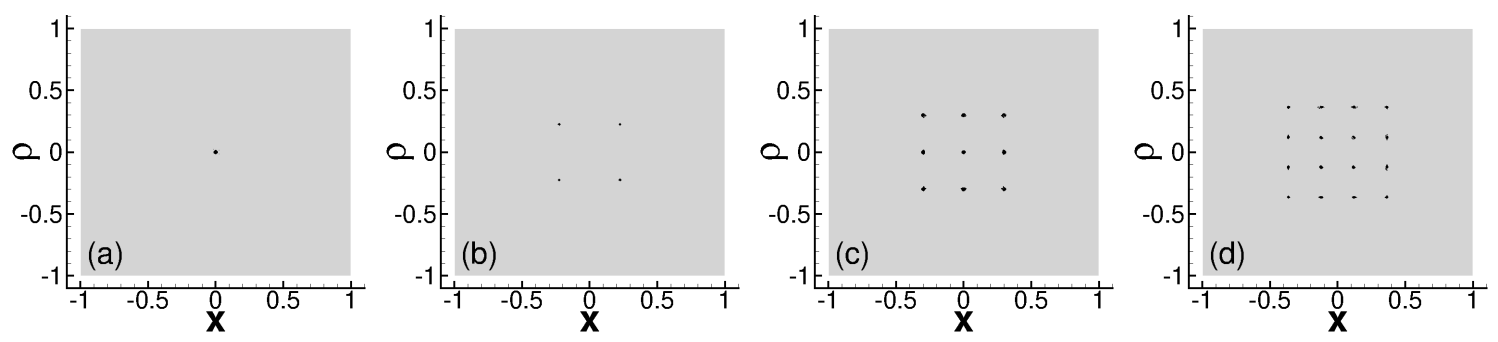

Figure 2: Contour plots corresponding to Fig. 1. (a) One-delta peak for $R=0.3 ; 30$ density contour lines ranging from 100 to 2800; (b) Four-delta peaks for $R=0.2$; 30 density contour lines ranging from 40 to 2000; (c) Nine-delta peaks for $R=0.15$; 30 density contour lines ranging from 30 to 500; (d) Sixteen-delta peaks for $R=0.1 ; 30$ density contour lines ranging from 30 to 450 .

peaks, respectively. This phenomena agree with the theoretical prediction in [5], demonstrating the effectiveness of the proposed method. To some extend, it is surprised that the FD method could produce such singular solutions. To the best of our knowledge, this would not be possible without using the proposed lifting method; see Eqs. (2.2) and (2.3).

\section{Conclusions}

We have proposed in the present work a new method to lift the solution space of the continuous Krause model, such that in each spatial direction $\delta$-singularities in the solutions are expected to transform into step functions, which can be handled directly by some nonlinear shock-capturing FD schemes. The key is to introduce a vector such that its divergence is equal to the density of the considered model. 
Then the lifted system in a splitting form can be obtained. Specially, we confined the study to the two-dimensional case to illustrate the idea. Since the lifted system is still hyperbolic as the original model, an upwind-biased splitting was performed in order to implement the chosen fifth-order WCNS. From the presented numerical results, we observed that the $\delta$-singularities in the solutions are well captured by the high-order FD scheme. Moreover, the numbers of $\delta$ peaks agree with the theoretical prediction, demonstrating the effectiveness of the proposed method. The method is certainly applicable for the considered model in higher dimension and also for some other similar models with $\delta$-singularities.

In a future work, one can consider the extension of the present method to solve the case with the Euclidean norm in definition (1.3), or the case with other computational domains as presented in [5]. In addition, as a new approach, some more basic properties of the lifting method need further investigations, especially in a more rigorous way.

\section{Acknowledgments}

This work is supported by the National Natural Science Foundation (Grant No. 11972370) and the National Key Project (Grant No. GJXM92579) of China.

\section{References}

[1] V. D. Blondel, J. M. Hendrickx and J. N. Tsitsiklis, On Krause's multi-agent consensus model with state-dependent connectivity, IEEE Trans. Autom. Control 54 (2009), 25862579.

[2] V. D. Blondel, J. M. Hendrickx and J. N. Tsitsiklis, Continuous-time average-preserving opinion dynamics with opinion-dependent communications, SIAM J. Control Optim. 48 (2010), 5214-5240.

[3] C. Brehm, M. F. Barad, J. A. Housman and C. C. Kiris, A comparison of higher-order finite-difference shock capturing schemes, Comput. Fluids 122 (2015), 184-208.

[4] C. Canuto, F. Fagnani and P. Tilli, An Eulerian approach to the analysis of rendez-vous algorithms. In: Proceedings of 17th IFAC World Congress. Amsterdam, Elsevier, 41 (2008), 9039-9044.

[5] C. Canuto, F. Fagnani and P. Tilli, An Eulerian approach to the analysis of Krause's consensus models, SIAM J. Control. Optim. 50 (2012), 243-265.

[6] G. Chen, W. Su, S. Ding and Y. Hong, Heterogeneous Hegselmann-Krause dynamics with environment and communication noise, IEEE Trans. Autom. Control 65 (2020), 3409-3424. 
[7] B. Cheng, Y. Chen and X. Deng, Solution to the Fokker-Planck equation with piecewiseconstant drift, Chin. Phys. Lett. 37 (2020), 060201.

[8] B. Cheng, Y. Chen, C. Xu, D. Li and X. Deng, Nonlinear Schrödinger equation with a Dirac delta potential: finite difference method, Comm. Theor. Phys. 72 (2020), 025001.

[9] X. Deng, M. Mao, G. Tu, H. Zhang and Y. Zhang, High-order and high accurate CFD methods and their applications for complex grid problems, Commun. Comput. Phys. 11 (2012), 1081-1102.

[10] X. Deng and H. Zhang, Developing high-order weighted compact nonlinear schemes, J. Comput. Phys. 165 (2000), 22-44.

[11] N. Guo, Y. Chen and X. Deng, A lifting method for hyperbolic equations with delta-singularities, J. Phys.: Conf. Ser. 1978 (2021), 012042.

[12] X. Hong and Y. Xia, Arbitrary Lagrangian-Eulerian discontinuous Galerkin method for hyperbolic equations involving $\delta$-singularities, SIAM Numer. Anal. Anal. 58 (2020), 125152.

[13] G. Jiang and C.-W. Shu, Efficient implementation of weighted eno schemes, J. Comput. Phys. 126 (1996), 202-228.

[14] S. Osher and C.-W. Shu, High-order essentially nonoscillatory schemes for HamiltonJacobi equations, SIAM J. Numer. Analy. 28 (1991), 907-922.

[15] E. Ozbenli and P. Vedula, Construction of invariant compact finite-difference schemes, Phys. Rev. E 101 (2020), 023303.

[16] C.-W. Shu and S. Osher, Efficient implementation of essentially non-oscillatory shockcapturing schemes, J. Comput. Phys. 77 (1988), 439-471.

[17] A. K. Tornberg and B. Engquist, Regularization techniques for numerical approximation of PDEs with singularities, J. Sci. Comput. 19 (2003), 527-552.

[18] E. Wedin and P. Hegarty, The Hegselmann-Krause dynamics for the continuous-agent model and a regular opinion function do not always lead to consensus, IEEE Trans. Autom. Control 60 (2015), 2416-2421.

[19] Y. Yang and C.-W. Shu, Discontinuous Galerkin method for hyperbolic equations involving $\delta$-singularities: negative-order norm error estimates and applications, Numer. Math. 124 ( 2013), 753-781.

[20] Y. Yang, D. Wei and C.-W. Shu, Discontinuous Galerkin method for Krause's consensus models and pressureless Euler equations, J. Comput. Phys. 252 (2013), 109-127.

[21] R.-P. Zhang, X.-J. Yu and G.-Z. Zhao, A new finite difference scheme for a dissipative cubic nonlinear Schrödinger equation, Chin. Phys. B 20 (2011), 30204. 